\title{
SOURCE PARAMETERS OF EARTHQUAKES, AND DISCRIMINATION BETWEEN EARTHQUAKES AND NUCLEAR EXPLOSIONS
}

\author{
By John B. Davies and Stewart W. Smith
}

\begin{abstract}
The first part of this study describes a technique by which the source parameters of an earthquake can be obtained from the spectrum of compressional waves. The source parameters defined are fault length, fracture velocity, and fault plane attitude. Two large, deep earthquakes are examined using this technique. The source parameters determined compare favorably with those obtained previously using different techniques. In the second section a method is proposed for discrimination between underground explosions and earthquakes. The technique utilizes the ratio of the spectrums of the two classes of events where the path of propagation is common to both. On the basis of the analysis of the SHOAL event and a nearby shallow earthquake it appears that the duration as determined from the spectral ratio is almost 10 times smaller for an explosion than it is for a comparable earthquake.
\end{abstract}

\section{INTRODUCTION}

The search for earthquake source parameters such as fault length, fracture velocity, and fault plane orientation has been continued vigorously in recent years with the introduction of new techniques which make use of the spectrum of body waves, surface waves, and free oscillations. In the first part of this paper, a technique will be described by which earthquake source parameters can be obtained from the spectrum of $P$ waves recorded at distant stations. Corrections for the effects of the path of propagation and the recording instrument must of course be made, and a deterministic model such as a propagating fracture must be assumed. In the second part of this study, it will be shown that source durations derived from the ratio of spectrums of earthquakes and explosions should be significantly different. Using the techniques developed here reasonable cavity dimensions can be obtained for underground explosions. Also, for one special case, perhaps the best example of an earthquake and an explosion in the same locality (SHOAL), it will be shown that the durations defined here are very much longer for earthquakes than they are for explosions of comparable size. On this basis it is suggested that the technique described here can be used to discriminate between earthquakes and explosions occurring in the same region.

The model taken for the earthquake sources will be that proposed by Ben-Menahem (1962) and Ben-Menahem et al. (1965) that is a moving-point source, with certain minor changes. The model for the explosive source will be that of a step pressure pulse on the inside of a spherical cavity (Sharpe, 1942).

\section{Analysis of Earthquake Spectrums}

From Ben-Menahem (1962), the surface displacements from a compressional wave in an inhomogeneous elastic half-space, due to a horizontal simple force $L e^{i \omega t}$ located at a depth $h$ below the free surface are

$$
\left[\begin{array}{c}
U_{r \alpha} \\
U_{z \alpha}
\end{array}\right]=\frac{L \cos \theta_{0}}{\pi \mu_{0} r}\left[\begin{array}{c}
2 P_{r}(h, r) \\
P_{z}(h, r)
\end{array}\right] e^{i \omega\left(t-t_{0}\right)}
$$


where there is no phase difference between radial and vertical components and where $r_{0}$ is the distance from source to point of observation

$\theta_{0}$ is angle between ray and force direction

$\mu_{0}$ is Lame constant

$t_{0}$ is travel time along seismic ray

$P_{r}, P_{z}$ are complex reflection coefficients.

Now at a certain time $t=0$, the source moves in the plane parallel to the free surface in the direction of the simple force ( $x$ direction), with a constant speed $U_{f}$, from $x=0$ to $x=b$, radiating as it moves. We assume the fracture velocity $U_{f}$ to be less than the compressional wave velocity $U_{p}$ at the depth $h$.

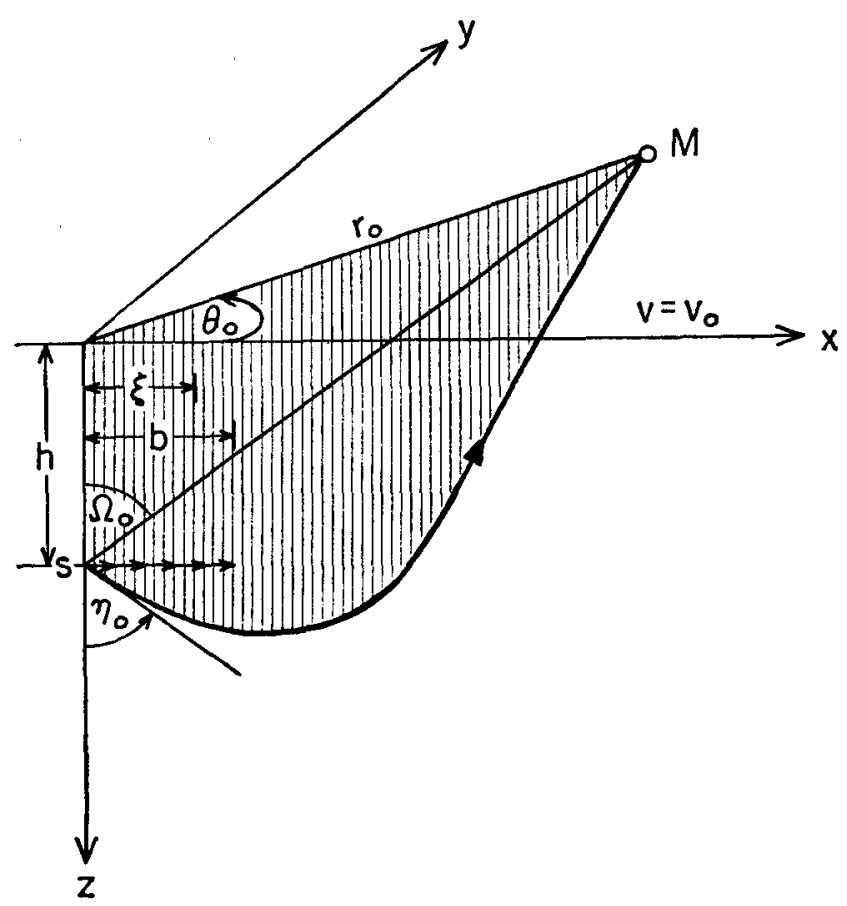

Fig. 1. A moving horizontal force in a medium with a weak velocity gradient (from Ben-Menahem, 1962).

The integrated far-field at the point of observation $M$ on the free surface of an inhomogeneous sphere (Figure 1) is then

$$
\left[\begin{array}{l}
U_{r \alpha} \\
U_{z \alpha}
\end{array}\right]=\frac{1}{b} \int_{0}^{b}\left[\begin{array}{l}
U_{r \alpha}(\xi) \\
U_{z \alpha}(\xi)
\end{array}\right] e^{-i \omega \xi / U_{f}} d \xi
$$

to the first order we have

$$
t_{0}(r)=t_{0}\left(r_{0}\right)-\frac{\xi}{U_{p}} \sin \eta_{0} \cos \theta_{0}+O\left(\frac{b}{r_{0}}\right)
$$

where $r$ is distance from point $\xi$ on line source to point of observation $\eta_{0}$ is the angle of the source between the vertical and the ray.

This differs from Ben-Menahem's result (1962) where he takes $U_{p}$ to be the com- 
pressional wave velocity at the free surface. From the diagram shown in Figure 1, it can be seen that the above is the correct expression. Then

$$
\cos \theta\left[\frac{P(r)}{r}\right]=\cos \theta_{0}\left[\frac{P\left(r_{0}\right)}{r_{0}}\right]+O\left(\frac{b}{r_{0}}\right) .
$$

Neglecting terms of order $\left(b / r_{0}\right)$ in amplitude and phase, we have

$$
\left[\begin{array}{c}
U_{r \alpha} \\
U_{z \alpha}
\end{array}\right]=\frac{L \cos \theta_{0}}{\pi \mu_{0} r_{0}}\left[\begin{array}{c}
2 P_{r}\left(h, r_{0}\right) \\
P_{z}\left(h, r_{0}\right)
\end{array}\right] \frac{\sin X}{\bar{X}} e^{-i X} e^{i(\omega)\left(t-t_{0}\right)}
$$

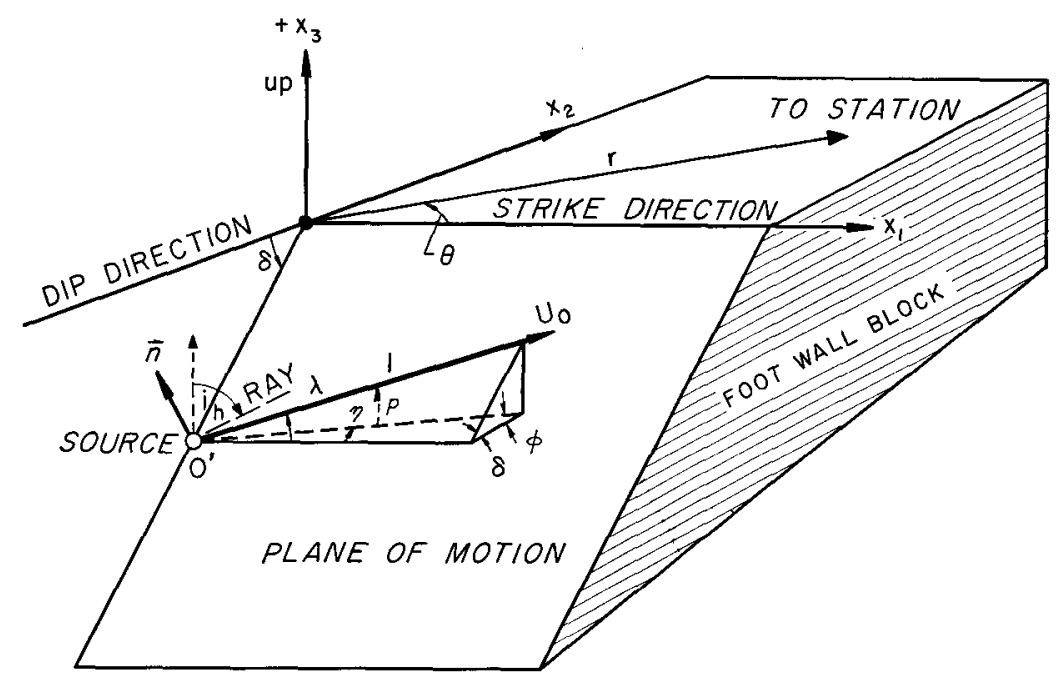

Fig. 2. Geometry of source elements and relative position of station on the free surface (from Ben-Menahem, Smith and Teng, 1965).

where

$$
X=\frac{\omega}{2}\left(\frac{b}{U_{f}}-\frac{b}{U_{p}} \sin \eta_{0} \cos \theta_{0}\right) .
$$

If the source moves along a general fault plane, as in Figure 2, we have

or

$$
X=\frac{\omega}{2}\left\{\frac{b}{U_{f}}-\frac{b}{U_{p}}\left[|\cos p| \cos (\theta-\eta)-\sin p \cot i_{h}\right]\right\}
$$

where

$$
X=\pi f\left[T_{f}-T_{p} g\left(\theta, \eta, p, i_{h}\right)\right]
$$

$$
\begin{aligned}
& g\left(\theta, \eta, p, i_{h}\right)=|\cos p| \cos (\theta-\eta)-\sin p \cot i_{h} \\
& \omega=2 \pi f
\end{aligned}
$$

fracture duration

$$
T_{f}=b / U_{f}
$$

compressional duration

$$
T_{p}=b / U_{p}
$$


It should be noted that the compressional duration $T_{p}$ is just the travel time of a $P$ wave along the fault. It does not imply that a process involving compression actually occurred over this interval of time. Now put $X=\pi f T$ and as we measure only the azimuth of the station relative to the source, we define $a$, the relative azimuth, as $a=\theta-c$, where $c$ is an angle to be obtained from the source parameter solution. Thus

$T=T_{f}-T_{p}\left[\{\cos c \cdot \cos \lambda+\sin c \cdot \sin \lambda \cdot \cos \delta\} \cos a \cdot \sin i_{h}\right.$

$$
\begin{aligned}
& -\{\sin c \cdot \cos \lambda-\cos c \cdot \sin \lambda \cdot \cos \delta\} \sin a \cdot \sin i_{h} \\
& \left.-\{\sin \lambda \cdot \sin \delta\} \cos i_{h}\right]
\end{aligned}
$$

where $T$ is termed the duration of the source.

So far, we have assumed the source to be a moving point force emitting a continuous wave of the form $L e^{i \omega t}$. As we are concerned with an earthquake source, we will consider a moving double-couple point source and not a moving point force. Also, as the information used is long-period, this double-couple point source can be taken to have a time dependence equivalent to a differentiated step function, which is a Dirac delta function, i.e., $L \delta(t)$. Implicit in this assumption is that each point of our distributed source exhibits a stress relaxation over a time short compared with the period of waves used in this analysis.

Thus, after removing the effects of path and recording, the spectrum of the displacement $P$ pulse can be represented by

$$
\left[\begin{array}{c}
U_{r \alpha}(f) \\
U_{z \alpha}(f)
\end{array}\right]=\left[\begin{array}{c}
c_{r} \\
c_{z}
\end{array}\right] \frac{\sin (\pi f T)}{\pi f t} e^{-i \pi f T} .
$$

The spectrum of the vertical component of the velocity time function is then

$$
V(f)=\frac{2 c}{T} \sin (\pi f t) e^{-i \pi f T}
$$

where $c$ is a real constant. This spectrum will only be known in a finite range of frequency.

Now if the source model proposed is approximately correct there should be a value of the parameter $T$ for which the theoretical spectrum is a best fit, in some sense, to the experimentally determined spectrum. Since it is the oscillation or ripple in the spectrum that contains the information about the parameter $T$, we should be able to estimate the period of this ripple by taking its spectrum, and thus determine $T$. This operation would return us to the time domain if it were not for the fact that in isolating the ripple prior to taking its spectrum we may modify the original spectrum by removing its mean value or subtracting a linear trend. Bogert et al (1962) used paraphrased terms to try to avoid confusion in applying time series terminology to operations on the spectrum of a time series. In their terminology the spectrum of a function of a spectrum would be referred to as a cepstrum, and the variable would be called the quefrency, which will have the dimensions of time. The information about this ripple can be obtained from the complex spectrum of the original spectrum. Alternatively, it could be obtained independently from either the real or imaginary part alone. Although its presence has not been explicitly accounted for, it seems clear that noise would seriously 
affect the results of such calculations. If, however, significant noise is present, the results for the duration $T$ determined independently from the real and imaginary parts of the spectrum would probably be inconsistent. We have computed $T$ in both ways and insisted on consistent results before accepting the data. Taking the real part of the velocity spectrum given in equation 10 and removing the mean we get

$$
R_{e}[V(f)-\bar{V}]=\frac{c}{T} \sin (2 \pi f T)
$$

Setting this function equal to zero for frequencies greater than $F$, that is outside the frequency range where we have data and taking the Fourier sine transform we obtain

$$
Q_{r}(q)=\frac{c}{T} \int_{0}^{F} \sin (2 \pi f T) \sin (2 \pi f q) d f
$$

and

$$
Q_{r}(q)=\frac{c F}{2 T}\left[\frac{\sin 2 \pi F(q-T)}{2 \pi F(q-T)}-\frac{\sin 2 \pi F(q+T)}{2 \pi F(q+T)}\right]
$$

This function has a peak at the positive quefrency equal to the duration $T$.

Similarly, taking the imaginary part of the velocity spectrum, removing the mean, and performing a Fourier cosine transform between the band limits, we have

$$
Q_{i}(q)=\frac{c F}{2 T}\left[\frac{\sin 2 \pi F(q-T)}{2 \pi F(q-T)}+\frac{\sin 2 \pi F(q+T)}{2 \pi F(q+T)}\right] .
$$

This function $Q_{i}(q)$ also peaks at a quefrency corresponding to the duration $T$. Thus, two values of the duration are obtained, with a mean duration taken from these two.

Alternatively, we could put the velocity spectrum in the form

$$
V(f)=\frac{2 c}{T} 2 i\left(1-e^{-i 2 \pi f T}\right)
$$

After removing the non-oscillating term

$$
V(f)=\frac{2 c^{\prime}}{T} 2 i e^{-i 2 \pi f T}
$$

Now, performing an exponential Fourier transform

$$
Q^{\prime}(q)=\frac{4 i c^{\prime}}{T} \int_{0}^{F} e^{-i 2 \pi f T} e^{+i 2 \pi f q} d f
$$

and taking the imaginary part

$$
\operatorname{Im}\left[Q^{\prime}(q)\right]=\frac{4 c^{\prime} F}{T}\left[\frac{\sin 2 \pi F(q-T)}{2 \pi F(q-T)}\right] .
$$

This function similarly has a peak at a quefrency equal to the duration $T$. 
The advantage of the first method over the exponential transform is that some qualitative feel for the errors inherent in these analyses can be obtained.

As all operations above have been linear, it is thus possible to design a linear filter to operate on the observed seismogram to obtain the identical time function which is called here the quefrency cepstrum. The word cepstrum is used however to emphasize that the time function obtained has to be interpreted differently from a normal seismogram. The peak at the duration $T$ indicates the length of the initial impulse and not that the maximum displacement or velocity occurred at this time. We will thus use the idea of cepstrum in this context only. We can now take the equalized spectrum of compressional waves, perform either of the above transform methods, and read off the duration from the quefrency cepstrum. This "duration" is a function of fault azimuth, take-off angle and actual source duration. If it can be measured at a number of stations, then it should be possible to fit these values to Equation 8 and determine the individual source parameters.

\section{Later Arrivals}

Seismograms often contain multiple compressional wave arrivals due to the $P$-pulse travelling different paths through the mantle or being reflected from discontinuities.

We assume that a later $P$-arrival from the source is received at a time $t_{d}$ after the first $P$-wave arrival. We also assume that this pulse has the same spectral form as the initial pulse. The velocity spectrum due to this arrival is

$$
V_{d}(f)=\frac{2 C_{d}}{T_{d}} \sin \left(\pi f T_{d}\right) e^{-i 2 \pi f\left(t_{d}+\left(T_{d} / 2\right)\right)}
$$

where $C_{d}$ is the amplitude of the arrival and $T_{d}$ is the duration of the arrival. This can be written

$$
V_{d}(f)=\frac{2 C_{d}}{T_{d}} 2 i\left[e^{-2 \pi i f t_{d}}-e^{-i 2 \pi f\left(t_{d}+T_{d}\right)}\right]
$$

Similar operations, as before, with Fourier transforms can yield the delay $t_{d}$ and the duration $T_{d}$. Clearly the above operation can yield estimates of source parameters only if the bandwidth available is adequate to resolve second arrivals from extended source effects. In cases where the delay between arrivals is comparable with the source duration the technique is not applicable.

\section{Two Examples}

The technique developed above is now applied to the $P$-wave spectra of two large, deep earthquakes. In both cases, and for all stations, the spectra are first equalized back to the source (Teng, 1966) by removing the effects of attenuation, crust, instrument, etc. on the amplitude and phase spectra. Due to the uncertainties in knowledge of crustal structures under most recording stations, and the time sample having a finite length, this equalization can only be done between 0.03 and $0.20 \mathrm{cps}$.

(A) The first example is a deep Brazil earthquake which has been analyzed for radiation patterns by Teng (1966). It occurred on November 9, 1963 (origin time 21:15:30.4 UT) with epicenter $\left(9.0^{\circ} \mathrm{S}, 71.5^{\circ} \mathrm{W}\right)$ and a magnitude $6 \frac{3}{4}$ to 7 as assessed by both Pasadena and Berkeley. Its focal depth determined from 31 pP-P and $26 s P-P$ measurements was $550 \pm 20 \mathrm{~km}$. The records used were from long-period instruments 


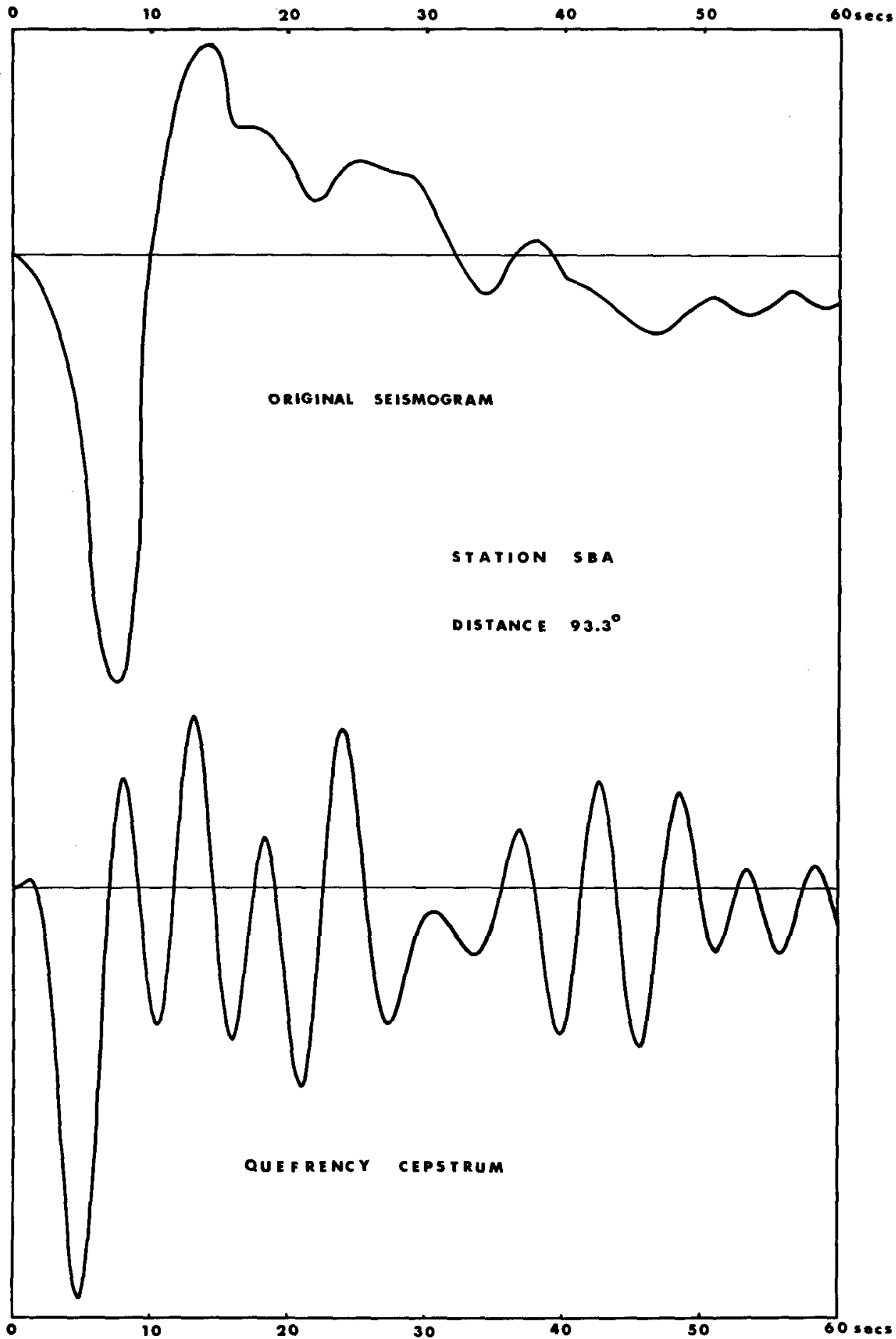

Fig. 3. Original seismogram and quefrency cepstrum of the $P Z$ phase from the Brazil earthquake for station SBA. 


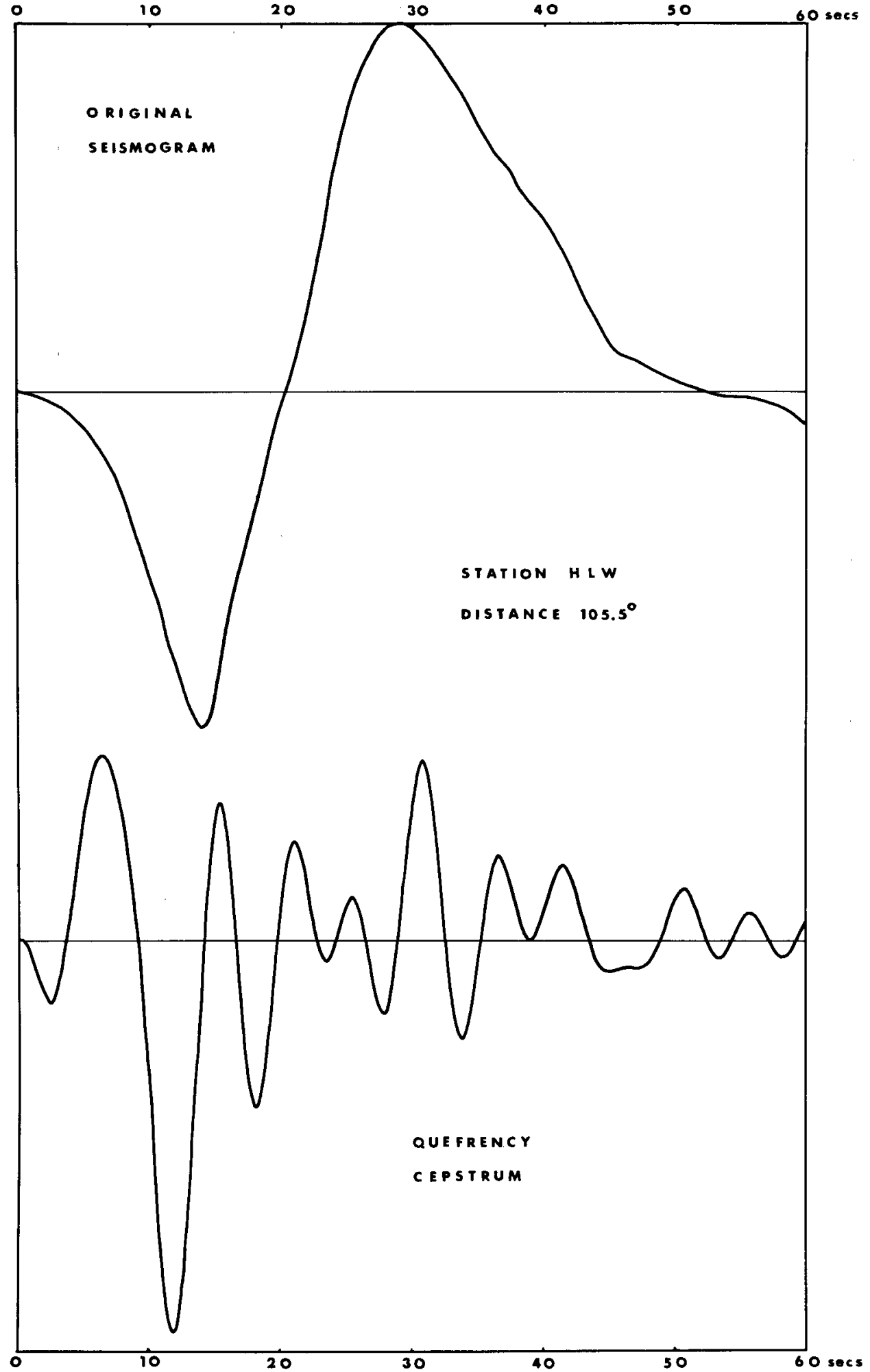

Fig. 4. Original seismogram and quefrency cepstrum of the $P Z$ phase from the Brazil earthquake for station $\mathrm{HLW}$. 
of the USCGS World-Wide Standardized Network. The time interval of Fourier analysis for all time records was 60 seconds and only the vertical component was used.

Figure 3 shows the seismograms of the $P Z$ phase and its associated cepstrum (the Fourier transform of the band-limited spectrum) for station SBA (azimuth: $179.7^{\circ}$; distance $93.3^{\circ}$ ). This cepstrum can be interpreted as consisting of an initial arrival with duration $4.8 \mathrm{sec}$ and another arrival at around 40 sec delay time. Due to noise and resolution effects, the exact value of delay and duration of the second arrival cannot be measured.

An interesting cepstrum is displayed, with the initial seismogram, in Figure 4. This is for station HLW (azimuth: $61.5^{\circ}$; distance: $105.5^{\circ}$ ). A first arrival of duration 12.0 sec, which is approximately twice as long as the average duration at all the other stations, is readily observable and the longer duration can be interpreted as due to the interaction of $P$ and $P c P$. A second arrival with a delay of 31 sec is readable though not absolutely certain. As the delay of $p P$ was greater than the length of time sample and was also readily observable later in the seismogram, this arrival has to indicate structure either in the upper mantle or at the mantle-core boundary.

TABLE 1

Data for the Brazil Earthquake

\begin{tabular}{lcccc}
\hline Station & $\begin{array}{c}\text { Relative Azimuth } \\
a \text { (degrees) }\end{array}$ & $\begin{array}{c}\text { Take-Off Angle } \\
i h \text { (degrees) }\end{array}$ & Duration (Mean) T(secs) & Residual (secs) \\
\hline BHP & 24.4 & 112.5 & 5.1 & -0.1 \\
BOG & 10.8 & 101.3 & 6.05 & +0.3 \\
STU & 319.2 & 152.9 & 5.4 & -0.3 \\
TOL & 313.6 & 149.0 & 5.6 & -0.3 \\
AAM & 11.4 & 135.0 & 4.55 & -0.5 \\
BOZ & 29.5 & 142.5 & 5.35 & +0.6 \\
KIP & 68.4 & 150.3 & 5.2 & +0.6 \\
OXF & 20.5 & 131.5 & 5.3 & +0.3 \\
AFI & 105.4 & 154.0 & 5.0 & +0.2 \\
BUL & 248.5 & 153.8 & 6.8 & +0.6 \\
PRE & 243.1 & 153.6 & 6.55 & +0.4 \\
\hline
\end{tabular}

Teng (1966), using amplitudes of $P$ and $S$ waves at stations surrounding the source, obtained radiation patterns for the earthquake and, correlating the observed pattern with theoretical patterns, obtained these fault parameters:

Strike Azimuth $c=170^{\circ}$

Fault motion direction $\lambda=270^{\circ}$

Dip $\delta=45^{\circ}$

We have obtained the duration at stations surrounding the source (Table 1) by the technique described above and, with these values have performed a least-squares fit to equation 8, using a technique as illustrated in Flinn (1965).

The following parameters were obtained:

Strike Azimuth $c=20^{\circ}$

Fault motion direction $\lambda=90^{\circ}$

Dip $\delta=45^{\circ}$

Fracture duration $T_{f}=7 \pm 0.5 \mathrm{sec}$

Compressional duration $T_{p}=1.7 \pm 0.5 \mathrm{sec}$

Using a compressional wave velocity of $9.8 \mathrm{~km} / \mathrm{sec}$ at the depth of $550 \mathrm{~km}$ we have

Fracture length $=27 \pm 5 \mathrm{~km}$

Fracture velocity $=3.8 \pm 0.7 \mathrm{~km} / \mathrm{sec}$ 
(B) The second example is that of a deep earthquake below the Banda Sea which Teng (1966) has also analyzed using radiation patterns. The event occurred on March 21, 1964 (origin time 03:42:19.6 UT) with epicenter $(6.4 \mathrm{~S}, 127.9 \mathrm{E}$ ) with magnitude $6 \frac{1}{4}$ to $6 \frac{3}{4}$. From $15 p P-P$ and $11 s P-P$ measurements the depth was determined to be $350 \mathrm{~km} \pm 20 \mathrm{~km}$. The durations, measured at stations surrounding the source have been obtained as in the first example and their values are shown in Table 2.

Teng (1966) in his analysis of this earthquake, using amplitudes to obtain radiation patterns, obtained the following parameters for this Banda Sea shock:

Strike azimuth $c=85^{\circ}$

Fault motion direction $\lambda=315^{\circ}$

Dip $\delta=84^{\circ}$

Fitting the durations obtained (Table 2) by a least-squares technique, the following values were obtained:

Strike azimuth $c=265^{\circ}$

Fault motion direction $\lambda=350^{\circ}$

Dip $\delta=60^{\circ}$

TABLE 2

Data for the Banda Sea Earthquake

\begin{tabular}{lcccc}
\hline Station & $\begin{array}{c}\text { Relative Azimuth } \\
a \text { (degrees) }\end{array}$ & $\begin{array}{c}\text { Take-Off Angle } \\
\text { ih (degres) }\end{array}$ & $\begin{array}{c}\text { Duration (Mean) } \\
\text { T(secs) }\end{array}$ & $\begin{array}{c}\text { Residuals } \\
\text { (secs) }\end{array}$ \\
\hline ADE & 197.9 & 131.5 & 9.3 & +1.0 \\
BAG & 17.6 & 128.4 & 6.1 & -0.3 \\
MON & 293.4 & 152.4 & 7.9 & -1.3 \\
MTJ & 345.7 & 137.4 & 8.3 & +0.2 \\
MUN & 158.2 & 130.5 & 5.5 & -0.8 \\
NHA & 45.2 & 129.9 & 6.7 & +1.4 \\
SEO & 1.1 & 137.8 & 7.0 & -0.4 \\
TAU & 202.2 & 135.5 & 9.6 & +1.1 \\
WEL & 222.7 & 141.8 & 8.4 & -0.7 \\
MAN & 17.9 & 126.9 & 6.2 & -0.2 \\
\hline
\end{tabular}

Fracture duration $T_{f}=7 \pm 1.2 \mathrm{sec}$

Compressional duration $T_{p}=4 \pm 1.1 \mathrm{sec}$

Using a compressional wave velocity of $8.8 \mathrm{~km} / \mathrm{sec}$ at $350 \mathrm{~km}$ depth, we have

Fracture length $=35 \pm 10 \mathrm{~km}$

Fracture velocity $=5 \pm 2.3 \mathrm{~km} / \mathrm{sec}$

Thus for two earthquakes, we have obtained important information about their source parameters by an alternative procedure to that used by others. Previously, point sources have been used to interpret body-wave seismograms and derive source parameters (see, for example, Teng, 1966). However, by using this idea of duration, more accurate source parameters can be obtained together with knowledge of the errors in the values so derived.

\section{Discrimination between Earthquakes and Explosions}

Sharpe (1942) has derived the far-field displacement time function for the case of a step pressure-pulse on the inside of a spherical cavity. He obtains

$$
D_{b}(t)=\frac{a^{2} \cdot p_{0}}{2 \sqrt{2} \cdot \mu r} \cdot e^{-\omega^{\prime} \tau / \sqrt{2}} \cdot \sin \left(\omega^{\prime} \tau\right)
$$


where

$a$ is the radius of the cavity

$p_{0}$ is amplitude of pressure pulse

$\omega^{\prime}=2 \cdot \sqrt{2} \cdot U_{p}^{*} / 3 a$

$\tau=t-r / U_{p}$

$\geqq 0$

$r$ is travel distance of wave

$U_{p}^{*}$ is compressional wave velocity at source.

The frequency spectrum of this displacement time function is given by

$$
\tilde{U}_{b}(f)=\omega^{\prime} /\left\{\left(\omega^{\prime} / \sqrt{2}+i \cdot 2 \pi f\right)^{2}+\omega^{\prime 2}\right\} .
$$

Between the frequency limits $\pm f_{\max }$, where

$$
f_{\max }=\sqrt{\frac{3}{2}} \cdot \frac{\omega^{\prime}}{2 \pi}
$$

this spectrum can be expanded in a complex Fourier series of period ( $4 f_{\max }$ ) i.e.,

$$
\widetilde{U}_{b}(f)=\sum_{n=-\infty}^{\infty} c_{n} \cdot e^{-i 2 \pi n f /}\left(\mathbf{4} \cdot \sqrt{\frac{3}{2}} \cdot \frac{\omega^{\prime}}{2 \pi}\right)
$$

Evaluating the coefficients $c_{n}$, we find

$$
c_{1}=\frac{1}{\sqrt{3} \cdot \omega^{\prime}}(0.84+0.02 i)
$$

with all other coefficients negligibly small relative to $c_{1}$.

Thus we can approximate the displacement spectrum from an explosive source by

$$
\widetilde{U}_{b}(f)=c^{\prime} \cdot e^{-i 2 \pi f T_{b}}
$$

where

$$
\begin{gathered}
c^{\prime}=\frac{a^{2} \cdot p_{0}}{2 \sqrt{2} \cdot \mu r} \cdot \frac{1}{\sqrt{3} \omega^{\prime}}(\cdot 84) \\
T_{b}=\frac{\sqrt{3} \pi}{4} \cdot \frac{a}{U_{p}^{*}}
\end{gathered}
$$

and where we have ignored the small imaginary contribution in the coefficient $c_{1}$.

To obtain some idea of the values that $f_{\max }$ and $T_{b}$ will possess in a nuclear explosion, let us take the Rainier event. This explosion was assigned a magnitude $M_{s}$ of 4.3 , and the radius of the cracking zone was calculated to be between 366 meters and 488 meters (Archambeau, 1965). As beyond the cracking zone is the purely elastic zone, we take the radius of this cracking zone to be the radius of the equivalent cavity in Sharpe's model. Assuming a local compressional wave velocity of $4 \mathrm{~km} / \mathrm{sec}$, we have an ex- 
plosion duration $T_{b}$ of .12 to .16 sec, with corresponding frequency limits $f_{\max }$ of 1.5 to $2.0 \mathrm{cps}$.

Consider now the case of an earthquake and an explosion occurring at or near the same location, emitting compressional waves that travel identical paths to a recording seismometer. By taking the ratio of the spectrum of the earthquake and the explosion, all path and measuring effects are removed. Thus, for first arrivals only, we have the spectral ratio

$$
R(f)=\frac{\tilde{U}_{e q}^{z}(f)}{\widetilde{U}_{b}^{z}(f)}=K^{\prime} \frac{\sin \left(\pi f T_{e q}\right)}{\pi f T_{e q}} e^{-i \pi f T_{e q}} / L^{\prime} e^{-i 2 \pi f T_{b}^{\prime}}
$$

where $K^{\prime}, L^{\prime}$ are real amplitudes

$T_{e q}$ is earthquake duration (as defined in eqn. 8)

$T_{b}$ is explosion duration (as defined in eqn. 21).

This ratio can now be expressed more conveniently as

$$
R(f)=\frac{K^{\prime}}{L^{\prime}} \frac{2 i}{\pi f T_{e q}}\left(1-e^{-i 2 \pi f T_{e q}}\right) / e^{-i 2 \pi f T_{b}} .
$$

In order to emphasize the oscillatory nature of this function in preparation for spectral analysis we remove the inverse frequency dependence, and obtain the final form

$$
R^{\prime}(f)=2 \pi f R(f)=\frac{4 i}{T_{\epsilon q}} \frac{K^{\prime}}{L^{\prime}}\left[e^{+i 2 \pi f T_{b}}-e^{-i 2 \pi f\left(T_{\epsilon q}-T_{b}\right)}\right] .
$$

Operating on this frequency ratio function $R^{\prime}(f)$ with positive and negative complex Fourier transforms, $\left(e^{+2 \pi i f q}\right.$ and $e^{-2 \pi i f q}$ respectively), will yield peaks in the cepstrum at quefrencies equal to $\left(T_{e q}-T_{b}\right)$ and $\left(T_{b}\right)$ respectively. It should be emphasized that this can only be done for frequencies less than or equal to the frequency limit $f_{\max }$.

Using durations obtained from stations surrounding the source, and using a least squares fit technique as before, it should be possible to obtain the source parameters for both earthquake and nuclear explosion.

\section{EXAMPLE}

The spectra of $P$ pulses from an earthquake-nuclear explosion pair were analyzed using the technique described above. The earthquake was the Fallon event of $20 \mathrm{July}$, 1962 , at a depth of $25 \mathrm{~km}$ and a body wave magnitude 4.4 . The nuclear explosion was the Shoal event of 26 October, 1962, at a depth of $0.4 \mathrm{~km}$ and a body-wave magnitude 4.9. The explosion had approximately the same epicenter as the earthquake. Four stations for this pair were analyzed. The separate $P$ phases are first isolated and selected from the original seismogram by using a rectilinear motion filter to obtain onset and interval times, (Archambeau and Flinn, 1965). Each isolated phase from the vertical seismogram is then filtered with a wide pass-band filter, and tapered with a cosine function over the latter 5 per cent of record. Only the initial $P$ phases were used in obtaining spectrum for the analyses. As the operations performed on each isolated phase were the same for both explosion and earthquake, any frequency effect of these operations will cancel on taking the spectral ratio.

Two stations of the four taken were amenable to this spectral technique and values for explosion duration and earthquake duration were obtained. The isolated initial $P$ phases for station BL-WV (distance $3200 \mathrm{~km}$ ) are displayed in Figure 5. Using a fre- 
quency band of $0.3 \mathrm{cps}$ to $1.0 \mathrm{cps}$, the transforms of the spectral ratio gave the nuclear explosion duration for this station to be 0.40 sec and the earthquake duration to be 2.35 sec.

The other station interpretable was DR-CO (distance $940 \mathrm{~km}$ ). Using a frequency band of 0.0 to $1.5 \mathrm{cps}$, the value of nuclear explosion duration at this station was 035 sec with an earthquake duration of $2.4 \mathrm{sec}$. The other two stations, CP-CL and DH-NY at distances from the source of $770 \mathrm{~km}$ and $3620 \mathrm{~km}$ respectively, were not interpretable

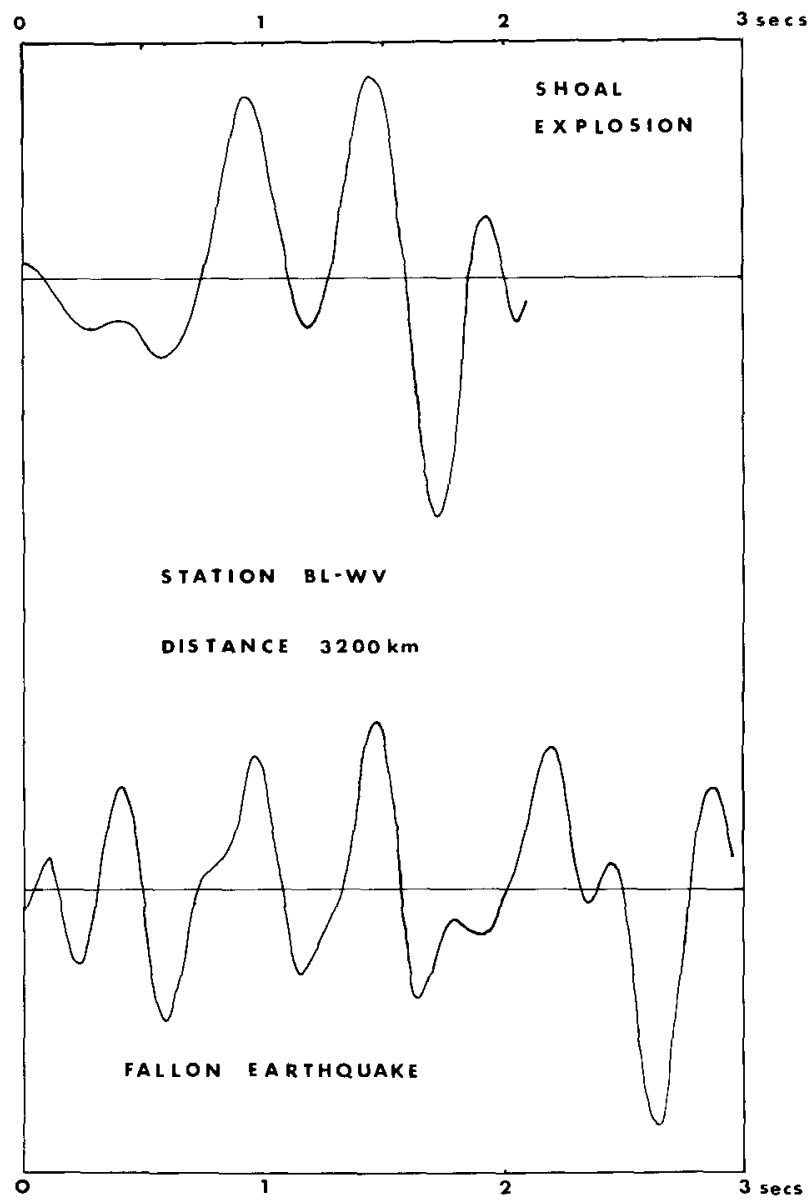

Fig. 5. Initial isolated $P$ phases from the Shoal nuclear explosion and Fallon earthquake recorded at Station BL-WV.

with this spectral technique. It is conjectured that this failure is due to excessively noisy seismograms or incorrect choice of the first arrival.

It is readily noticeable that the duration of the explosive source found here is markedly longer than that of Rainier. E. A. Flinn (personal communication) informs us that as the yield ratio of Shoal to Rainier is 6.8 , and as one would expect the radius of the cracked zone to go up as the cube root of the yield, the "duration" would go up in the same way. However, the duration of Shoal is greater than of Rainier by a factor $0.4 / 0.15=2.6$, which is close to the square root of 6.8 ; Flinn conjectures that preexisting jointing and zones of weakness may affect the relationship. 
Another possible reason for a longer observed duration for Shoal is that the arrival may be contaminated by $p P$ which, for an explosion depth of $0.4 \mathrm{~km}$ in material of $P$ velocity $4 \mathrm{~km} / \mathrm{sec}$, has a delay of about 0.2 secs. Thirlaway (1966) has also postulated such arrivals.

\section{Discrimination}

Similarly we can obtain spectral ratios for compressional pulses from two nuclear explosions from the same location and recorded at the same station. We have i.e.,

$$
R_{b}(f)=\frac{L_{1}}{L_{2}} e^{-i 2 \pi f\left(T_{b_{1}}-T_{b_{2}}\right)}
$$

for $f$ between the appropriate limits.

As the resolution of this method is little better than 0.05 sec, the durations $T_{b_{1}}$ and $T_{b_{2}}$ will have to differ by at least this amount in order to obtain this difference by the Fourier transform method.

If the spectral ratio of $P$ pulses from two earthquakes is taken, we have:

$$
R_{e q}(f)=\frac{M_{1}}{M_{2}} \frac{\left(1-e^{-i 2 \pi f T_{1}}\right)}{\left(1-e^{-i 2 \pi j T_{2}}\right)} .
$$

That is, after removing the non-oscillating term,

$$
R_{e q}(f)=\frac{M_{1}}{M_{2}}\left(-e^{-i 2 \pi f T_{1}}+e^{-i 2 \pi f T_{2}}-e^{-i 2 \pi f\left(T_{1}+T_{2}\right)}+\cdots\right)
$$

where longer duration terms have been ignored. Fourier transform of this ratio will give the durations $T_{1}$ and $T_{2}$.

We now wish to use this knowledge of the difference in spectrums between nuclear explosions and earthquakes in order to discriminate between them. Theoretically, if the spectrum of an initial $P$ pulse from a suspicious seismic event is compared, by the spectral ratio method, with a standard earthquake or nuclear explosion spectrum from the same region, then it should be possible to obtain the nature and duration of the event as outlined here. There will of course be serious problems with low level signals in which noise becomes a significant factor. As the technique of using the spectral ratio of two events has a sound theoretical background, however, it should be possible to derive a more advanced technique for estimating durations when noise is taken into account. The important result to note is that the duration, as defined above, of earthquakes is from two to ten times larger than that of nuclear explosions of similar magnitudes.

The relationship between the discrimination techniques described here and other methods currently in use can only be mentioned briefly. An evaluation of the effectiveness of our technique compared with these others cannot be made in this paper because of the limited number of examples presented here. Thirlaway (1966) summarized the apparent difference in reverberation time or "complexity" between earthquakes and explosions. The time interval over which continuing $P$ wave radiation arrives as measured by this array process is significantly greater than the durations measured here. They are in fact too large to be accounted for by radiation from different parts of an extended source region, and most probably can be explained by conversion of $S$ - to $P$-wave types in the vicinity of the source. Thus, there is no direct relation between the "complexity" and the "duration" of seismic sources. Liebermann et al. (1966) find consistent differences between body-wave and surface-wave magnitudes calculated for 
earthquakes and explosions. The effect is one of relatively greater excitation of longperiod surface waves by earthquakes than by explosions of the same body-wave magnitude. The partition of energy between long and short periods, between long and short wavelengths, and between shear and compressional wave types is the basic ingredient of their technique. The technique used in this paper makes use only of the $P$ wave, in a narrow band of frequencies, and basically depends on detecting interference effects in the radiation from different parts of a distributed seismic source. Therefore it does not appear that there is any direct relationship between this method and either of the two mentioned above.

\section{Conclusions}

A technique for the determination of earthquake source parameters from the spectrum of compressional waves has been described, and several examples of large deep earthquakes have been analyzed. The source dimensions, attitudes, and rupture velocities obtained compare favorably with those previously determined using different methods. A parameter termed "duration" is defined in terms of a specific model of a propagating source. The analysis of the SHOAL nuclear event and a shallow earthquake in the same location indicated that there may be a great difference in the duration of earthquakes and explosions. The data on which this conclusion is based is limited, and it is difficult to assess the importance of source depth in the determination of duration. In spite of these limitations we suggest that the duration, as defined here, can be used to discriminate between earthquakes and explosions occurring in the same region.

\section{ACKNOWLEDGMENTS}

It is a pleasure to thank Professors Charles Archambeau and James Brune for many helpful suggestions. The use of data from Dr. Ta-Liang Teng and critical review of the manuscript by Dr. Edward Flinn are gratefully acknowledged.

This research was supported by the Air Force Office of Scientific Research, Office of Aerospace Research, United States Air Force, under AFOSR contract AF-49(638)-1337. During a period of this research, one author (John B. Davies) held an International Business Machines scholarship.

\section{REFERENCES}

Archambeau, C. B. and E. A. Flinn (1965). Automated analysis of seismic radiation for source characteristics, Proc. of $I E E E$, 53, 1876-1884.

Archambeau, C. B. (1965). Elastodynamic Source Theory, Ph.D. Thesis, Calif. Inst. Tech.

Ben-Menahem, A. (1962). Radiation of seismic body waves from a finite moving source in the Earth, J. Geophys. Res. 67, 345-350.

Ben-Menahem, A., S. W. Smith and T. L. Teng (1965). A procedure for source studies from spectrums of long-period seismic body waves, Bull. Seism. Soc. Am. 55, 203-235.

Bogert, R. P., M. J. Healy and J. W. Tukey (1962). The quefrency analysis of time series for echoes, Time Series Analysis. Wiley, New York, p. 209.

Flinn, E. A. (1965). Confidence regions and error determinations for seismic event location, Rev. Geophys. 3, 157-185.

Sharpe, J. A. (1942). The production of elastic waves by explosion pressures, Geophysics, 7, 144154.

Teng, T. L. (1966). Body wave and earthquake source studies, Ph.D. Thesis, Calif. Inst. Tech.

Thirlaway, H. I. S. (1966). Interpreting array records: explosion and earthquake $P$ wavetrains which have traversed the deep mantle, Proc. Roy. Soc. A., 290, 385-395.

Contribution No. 1495, Division of Geological Sciences

Setsmologtcal Laboratory

California Institute of Technology

Pasadena, California

Manuscript received December 18, 1967. 